\title{
Interculturalidad, universidad y movimientos sociales latinoamericanos: ideas desde la frontera norte
}

\author{
Miguel Rábago Dorbecker \\ Doctorado en Derecho pela Universidad de Salamanca, España. \\ Profesor en la Universidad Iberoamericana, Cidade do México, México, México \\ miguel.rabago@ibero.mx
}

\begin{abstract}
Resumen
El ascenso social por la vía de la Educación Superior, parece ser una promesa difícilmente cumplida en el actual panorama de América Latina. La educación bancaria de las críticas de Freire e Illich ha llegado para quedarse, lo que deja la paradoja abierta entre la Universidad como fábrica y la Universidad como lugar de politización. ¿QQué queda de las lucha por la inclusión de sujetos subalternos a las universidades? ¿Más aún, para que integrarlos? La propuesta que subyace es hacer frente a esta paradoja, no sólo incluir, sino interculturalizar y decolonizar la universidad.
\end{abstract}

Palabras clave: educación superior, movimientos estudiantiles, educación intercultural, educacional en la América Latina.

\section{1. Élite y educación: el fin de la promesa de la universidad como legitimadora de la movilidad social}

$\mathrm{E}$ 1 terreno que ocupaba la educación superior en América Latina, como forma de ascenso social, así como instrumento de incorporación de grupos subalternados por el proceso de formación de ciudadanía liberal, parece convertirse en una promesa incumplida del sistema de modernidad capitalista. Aún en los terrenos de las escuelas más elitistas europeas, bajo cuya sombra se planteó el sistema universitario liberal de América Latina, encontrábamos el lugar imaginado de la movilidad social, aún en un continente tan poco propenso para estas cuestiones. Tony Judt (2010, p. 111) comenta en su excelente libro de memoria The Memory Chalet, cómo un inglés de origen judío y de clase media baja, pudo abrirse camino no sólo para llegar a Cambridge y la École Normale Supérieure, sino a convertirse en uno de los historiadores más renombrados de su generación. ¿Podría ahora un joven o una joven latinoamericana de clase media baja y de etnicidad indígena llegar a los máximos honores académicos en México, Colombia, Brasil o Argentina? Los cambios en el sistema de educación superior, así como en las formas de producción y de concentración del mercado, hacen de las acreditaciones universitarias y especializaciones de posgrado, cada vez más necesarias para la entrada de personas que proviene de clases subalternas, pero a la vez cada vez más flexibles para aquellas que provienen de las clases ultra privilegiadas. De hecho la propia aspiración 
de objetividad de las universidades de élite hacen de las mismas instrumentos legitimadores importantes de las propias clases dominantes y sólo residualmente un vehículo de incorporación de clases menos privilegiadas, para cuyo acceso tienen que librar grandes barreras (Rothbart, 1970, p. 164). En términos étnicos, este proceso puede ser considerado como de blanquitud (Echeverria, 2010, p. 57). El acceso a las universidades de élite cada vez está más marcado por los privilegios de clase, más que por un rígido sistema de control meritocrático. O más bien podría, como señala Iván Illich, considerarse que este es el currículum oculto del sistema en el que sólo importa: "que los estudiantes aprendan que la educación es valiosa cuando se adquiere en la escuela a través de un proceso graduado del consumo; que el grado de éxito que disfrutará el individuo en la sociedad depende de la cantidad de conocimientos que consume, y que los conocimientos sobre el mundo son más valiosos que los conocimientos adquiridos en el mundo" (Illich, 2006, p. 127). Freire ubica de modo los diferentes sistemas de domesticación e inscripción de la autoridad a través de la educación bajo la denominación de la educación bancaria (Freire, 2008, p. 75)

Sin embargo, aún con este currículo oculto y de educación bancaria, la educación superior no sólo es un medio de movilidad social, sino también de formación política para muchas personas. Las fuertes movilizaciones protagonizadas por los estudiantes universitarios latinoamericanos, que siguen ocupando un lugar privilegiado en las manifestaciones sociales y en las exigencias de cambios políticos-económicos. La geografía de la disidencia universitaria, pasa por las grandes manifestaciones por una educación superior gratuita y de calidad en Chile, Colombia y Puerto Rico, así como el movimiento Yo Soy 132 y Más de 133 en México. ¿Cómo han incorporado estos movimientos una reivindicación hacia la educación intercultural y la modificación de las prácticas autoritarias, patriarcales y racistas en la educación superior latinoamericana? Para responder esta pregunta hay que hacer un recuento de la relación entre universidad y movimientos sociales y la diferencia entre movimientos desde la universidad y aquellos enfocados en el cambio en la universidad.

De manera contradictoria, la universidad funciona como distribuidora del estatus elitista, a la vez que es un centro de discusión política fundamental. Si bien la movilidad social prometida por la universidad es, más que una ruptura en el sistema de clases, una reinscripción y confirmación de la misma. Por otro lado también es un lugar de encuentro y de intercambio de movimientos políticos radicales. En otras palabras, la universidad parece servir a dos amos: aquellos que cuestiona el sistema de clases, así como aquellos que la refuerzan (Rothbart, 1970, p.
162). Esta misma frase se puede extender al sistema de exclusión étnica, por un lado la universidad sirve para resguardar el eurocentrismo tradicional y la creación de una ciudadanía liberal única, como también es un lugar donde dichos conceptos se ponen a prueba y se desestabilizan.

\section{Universidad y cambio social. Movimientos para el cambio desde la universidad, para la universidad y para y desde la universidad}

La marcada tendencia histórica a relacionar los movimientos estudiantiles con las experiencias de 1968 es una más de las formas en las que se le otorga un privilegio histórico a ciertos eventos, en especial a los acontecidos en el mayo francés y las movilizaciones estudiantiles estadounidenses, minimizando las ocurridas en Latinoamérica, Europa del este y en Japón. Uno de los principales productos de este "sesentayochismo"es la minimización de las posteriores movilizaciones estudiantiles por muchos de los líderes de tales movimientos. En México la ruptura entre los antiguos estudiantes del 68 mexicano y diversas luchas sociales quedó, patente con la incorporación o cooptación de algunos líderes por parte de gobiernos priistas, los partidos de izquierda, la burocracia universitaria o por un abandono radical de antiguas posturas, para abrazar posturas de derecha y el neoconservadurismo. Al igual que en Alemania y Francia, en muchos casos los y las protagonistas de la generación del 68, ocuparon prominentes puestos políticos y universitarios desde finales de la década de los ochenta hasta nuestros días. Este periodo coincide con la adopción de las reformas estructurales neoliberales, sobre todo en un incremento feroz en la competencia por las plazas en la educación superior, privatización de la misma e incremento considerable de cuotas. Dichas reformas fueron implementadas con la muy cuestionable complicidad de los partidos tradicionales de izquierda e inclusive con la participación de algunos arrepentidos y no tan arrepentidos, miembros destacados del "sesentayochismo". Uno de los aspectos más contradictorios, es que esta participación de una parte de la generación del sesenta y ocho en los gobiernos y como autoridades universitarias durante el esquema privatizador de la universidad, los hacía aliados en el contexto latinoamericano de los represores. El "consenso" de Washington implicó que lideres arrepentidos de la nueva izquierda y oligarquías demasiado cercanas a los represores militares, compartieran responsabilidades respecto a los esfuerzos privatizadores y de medición y control en la 
educación superior. Como señala Martín Almada en el caso de Paraguay, la conversión en valor de cambio de la educación superior o sus mercantilización, fue parte de la doctrina de seguridad nacional implementada durante las dictaduras sudamericanas, esto en un marco de un aumento significativo de las tasas de ingreso (Almada, 2013, p. 288). En 2008, al cumplirse cuarenta años de los movimientos del 68, muchos de sus líderes se vanagloriaban en los logros obtenidos, casi siempre auto-referenciales y acríticos, mientras que se lamentaban de la "apatía" existente en los y las estudiantes actuales. Sin embargo, pocos años después un segundo movimiento estudiantil despertaría en América Latina, cuestionando todos los supuestos de los líderes históricos.

El "sesentayochismo" presentaba una forma de movilización, que si bien exigía cambios en el sistema superior educativo, esto resultaba tangencial en el objetivo mayor de la transformación general de la sociedad. Sin embargo, los movimientos estudiantiles que se gestaban en Estados Unidos, tenían no sólo un pie puesto en una transformación radical de las relaciones de género y raciales en dicho país, sino que mantenían una mayor congruencia entre la demanda de cambios externos e internos al sistema educativo universitario que perseguían. Dos ejemplos radicales de dicha congruencia fueron la huelga en la Universidad Estatal de San Francisco y la Universidad de Berkeley entre 1968 a 1969 por el Third World Liberation Front y las acciones militares del grupo que nació a raíz de las protestas estudiantiles conocido como The Weather Underground. Llama la atención que dentro de uno de los sistemas universitarios más elitistas y de alto costo, también con mayor participación de inversión privada, se den este tipo de manifestaciones de cambio radical.

Por lo que se refiere a la formación del Third World Liberation Front, este nace en un primer momento en el proceso que llevaría a una primera huelga en la Universidad Estatal de San Francisco en 1968. Tal organización tenía varias particularidades, comenzando con su configuración y siguiendo por sus demandas. En su configuración mostraba un carácter de frente amplio de organizaciones estudiantiles que apuntaban hacia la diversidad étnica permitida a raíz de los primeros esfuerzos del movimiento por los derechos civiles, de eliminar barrearas raciales y crear también acciones positivas para garantizar la entrada de diversas personas de distintas etnicidades a la Universidad. El Third World Liberation Front era entonces heredero y continuador de tales reivindicaciones y se formó por organizaciones estudiantiles que incluían aquellas formadas por afroamericanos (Black Student Union), latinos y latinas (Latin American Student Organization), asiáticos (Asian American Political Alliance), filipinos (Filipino American Collegiate Endeavor) e indígenas nor- teamericanos (Native American Student Union).Sus demandas incluían una vinculación expresa con el movimiento por los derechos civiles, al exigir mayores cuotas de estudiantes pertenecientes diversos grupos étnicos y también cambios en el esquema curricular. El movimiento estudiantil creado en la Universidad Estatal de San Francisco, tendría dos características esenciales, la creación de un bloque histórico en términos gramscianos entre organizaciones estudiantiles de diversas etnicidades y un énfasis en un cambio en la configuración étnica interna en la Universidad. Dicho movimiento culminó con la creación del departamento de estudios étnicos en dicha universidad.

Un año después, un movimiento similar realizaría una huelga en la Universidad de California en Berkeley, también en San Francisco. Las estrategias y el resultado serían similares, formación de un bloque de organizaciones estudiantiles de orígenes étnicos distintos y la respuesta institucional de la creación de un centro de estudios étnicos en la universidad. En ambos casos, los departamentos y centros de estudios étnicos fueron creados a través de una movilización, violentamente reprimida por la policía en ambos casos (Anderson, Gonzalez y Lee, 2006, p. 1906).

Finalmente los denominados Weather Undergorund que funcionó como una grupo de guerrilla urbana creado durante los tumultuosos movimientos estudiantiles de los sesenta, que también compaginaban las protestas en contra del sistema militar norteamericano con exigencias de igualdad racial. De hecho gran parte de los miembros de dicho grupo compacto, pertenecían con anterioridad a un grupo denominado Students for a Democratic Society. Lo que resulta en realidad muy llamativo de este grupo, no es su radicalidad u opción por la violencia, sino el sentido de solidaridad de universitarios y universitarias de clase media, media alta y blancos, que optan por aliarse con los movimientos afroamericanos más radicales. En este sentido, la apelación anti-racista caracteriza en gran medida a este grupo (Berger, 2005).

Estos ejemplos muestran como otras formas de protestas estudiantiles se separan del tradicional "sesentayochismo" que se quedó en un momento de transformación fundamental, pero cuyos líderes y consignas fueron rápidamente incluidas dentro del sistema de reproducción capitalista del Estado y de la educación. Sin embargo, el sesenta y ocho francés inauguró una forma de protesta que se vería replica$\mathrm{da}$ en las protestas estudiantiles que tuvieron mayor efectividad después del mayo del sesenta y ocho. Estas formas fueron la del desplazamiento del acto de protesta/acontecimiento de los estudiantes vía esquemas de empatía y solidaridad a otros movimientos sociales, logrando un cuestionamiento mucho más amplió de las formas sociales del régimen político.Este esquema se repitió en la transformación de una protesta 
en contra del candidato presidencial y actual Presidente de México por un grupo de estudiantes en la Universidad Iberoamericana, a extenderse a través de adhesiones flexibles y variantes a otras organizaciones estudiantiles y sociales (Barrios, 2012). Lo mismo aconteció con las protestas estudiantiles chilenas, que ahora se plantean en términos de reforma del estado a través de un acuerdo de reforma constitucional con los partidos políticos de la concertación. Estos esquemas de repetición, solidaridad o de redefinición y transformación de los movimientos estudiantiles implican el riesgo de que las exigencias originarias se pierdan o sean secuestradas o reinterpretadas por otros grupos sociales. Por lo que se refiere a exigencias que se centran en el espacio interior/exterior de la universidad, el riesgo es aún mayor, ya que la capacidad de mantener exigencias de modificaciones a los criterios de ingreso, relaciones estudiantiles, cambios en los programas, tasas y otras reivindicaciones, ante una apelación a un mayor enfoque en problemas más amplios de la sociedad, parece insostenible. Aquí hay que dividir entre movimientos estudiantiles que tenían una reivindicación política nacional o internacional en un primer momento y aquellos que se concentraban en temas delimitados a la Universidad. En este primer grupo encontramos a los movimientos del 68 y el Yo Soy 132, un ejemplo de los últimos serían el protagonizado por los estudiantes chilenos y el de las universidades en San Francisco en 1968 y 1969. Estos dos últimos movimientos, a través de una línea de acción clara, pudieron mantener las reivindicaciones de cambios dentro de la universidad, a la vez que apelar a cambios políticos fuera del ámbito universitario.

\section{Diversidad etnoracial y de género en las Universidades Latinoamericanas}

La nueva ola de manifestaciones por parte de sectores universitarios se da en el marco de políticas de inclusión étnico y racial y de género en las universidades latinoamericanas a través de una batería de recursos. Una referencia es el sistema de cuotas de las universidades brasileñas y los criterios de selección en base a diversidad étnico-racial en EUA, que afectan a un grupo considerable de latinoamericanos y latinoamericanas. Otra vertiente de más reciente aparición son las universidades multiculturales, pluriculturales e interculturales e incluso universidades indígenas o latinoamericanas. A estas dos opciones más inscritas en el debate multicultural de reconocimiento, se le añade un sistema mucho más añejo de becas espe- ciales para ciertos grupos: mujeres, indígenas, población afro-descendiente y hasta muy recientemente tímidas propuestas de sistemas de becas para personas del colectivo LGTTTBI. Todas estas opciones por crear sistemas de incorporación a un sistema educativo de corte liberal de características de blanquitudpatriarcal-heteronormativas llevaba a procesos, ya sea de uniformidad o de segmentación, que representan apenas algunas atenuantes al tradicional sistema de ingreso y permanencia en el sistema universitario. Sin embargo, las opciones por la creación de un sistema independiente de educación indígena, o un sistema de financiamiento especial o cuotas, implican opciones de políticas etno-raciales y de biopoder distintas.

En un primer lugar el sistema sigue siendo occidentalizante y sujeto a todo tipo de técnicas de una sociedad disciplinaria, en la que todos las personas no deseadas, pasan por un proyecto de integración en una subjetividad/ciudadanía bien determinada. En el caso de Brasil, la negación de las diferencias raciales dentro de la democracia racial, en el caso de México la ficción del proyecto de educativo de José Vasconcelos de un mestizaje a través de la educación y la creación de la raza de bronce. En este último caso, la creación de una subjetividad nacional centraba dicho proyecto en una des-indianización en la que se optaba por un mestizaje que más bien reflejaba "la vía criolla" (Vieyra, 1996, p. 54). Este proceso de aprendizaje y aculturación, heredaba los preceptos de la educación instaurada en los periodos liberales, bajo la cual el acceso a la universidad a través de las carreras liberales como el Derecho y la Medina confirmaban tanto la desindianización como la prevalencia hegemónica de la educación eurocéntrica.

Si bien dichos proyectos por lo menos no aparecen insertos en el discurso oficial, el nuevo multiculturalismo neo-liberal como lo denomina Charles Hale, parece que contiene apenas superficialmente los mismos propósitos disciplinarios (Hale, 2005). Por otro lado, la posibilidades segregacionistas de la educación indígena, no son solo ideas del conservadurismo liberal que pretende imponer el pensamiento único y el monolingüismo, sino también una posible instrumentalización por parte del sistema de dominación social, para mantener a los pueblos indígenas fuera de las universidades de élite y la consecuente estructura económica montada en torno a ellas. Este último aspecto también condenaría a las universidades indígenas a espacios no deseables para la movilidad social y arraigaría más el sentido de élite de las universidades tradicionales. No obstante, en los grandes sistemas universitarios públicos latinoamericanos como el brasileño, el argentino y el mexicano, la capacidad de intervención en proyectos interculturales o multiculturales a través de una plétora de sistemas de regulación, de estímulos y estandariza- 
ción de pruebas y programas de estudios, permite un monitoreo permanente que mina considerablemente la capacidad de autonomía de dichos programas o universidades.

El sistema de cuotas también muestra algunos problemas, en primer lugar, la re-deificación de las universidades de élite como acreditadoras de prestigio para la movilidad social. Por otro lado, la clara aculturación de currículo y la desvinculación de las comunidades. Los sistemas de cuotas no son nuevos, por ejemplo la Unión Soviética mantenía un sistema de cuotas en función del origen de clase de las personas que aspiraban a la Universidad, favoreciendo a hijos e hijas de trabajadores antes que a los que provenían de familias de graduados universitarios. El posterior desarrollo realizado en función de criterios de género y etnicidad y sólo en algunos casos, tímidamente bajo criterios de clase, realizado vía judicial en Estados Unidos, también marcaría el uso de dichas cuotas en la educación superior en América Latina. No obstante el debate se encuentra muy lejano de su conclusión.

\section{Movimientos estudiantiles y las reivindicaciones de clase, género y etnicidad}

Se puede decir que dentro de la triada de estructuras de poder discriminatorio de etnicidad, género y clase, la cuestión de clase ha sido la que lleva un mayor grado de representación en las exigencias de los movimientos estudiantiles. En el contexto de la mayor universidad pública mexicana, la huelga que estalló en la Universidad Nacional Autónoma de México durante poco más de nueve meses entre 1999 y 2000 , fue detonada por reformas en el sistema de pagos, pero también al sistema de permanencia y sobre todo de ingreso a dicha universidad. Previamente había diferentes movilizaciones relativas a la implementación de ciertos sistemas de medición y estandarización de criterios de ingreso y egreso, concentrados en el CENEVAL. ${ }^{1}$ Es decir, la estandarización, modelos nacionales de becas y sistemas de evaluación privatizados, diseñan toda una red de instituciones privadas y públicas o de operación simultánea público/privada que mantienen a todas las instituciones educativas superiores bajo un control administrativo homogenizado pero descentralizado.
Una cuestión que resulta fundamental es que el movimiento estudiantil conocido como Yo Soy 132 y Más de 133 en México apuntó sus demandas hacia cuestiones relativas al modelo económico, la educación gratuita, de manera que se hermanaban con los movimientos estudiantiles en Chile, Puerto Rico y Colombia, además de mantener una larga historia de movimientos estudiantiles en México desde el 68 hasta la fecha. A estas demandas de carácter más tradicional, se la añadió un importante reclamo a los medios de comunicación y a su democratización. Sin embargo, el tema relativo a desigualdades étnicoraciales o de género parecía encontrarse subsumido, como sucede con frecuencia en distintos movimientos sociales,en una amplía denuncia de la desigualdad en base a términos de posición de clase. Hay que señalar la amplía relación de algunos sectores del movimiento estudiantil con movimientos campesinos e indígenas. Dicha relación parecía acercarse mucho a la relación que guarda el movimiento de víctimas de la violencia generada por el conflicto armado con ciertos representantes de causas de los pueblos indígenas como el Ejercito Zapatista de Liberación $\mathrm{Na}-$ cional - EZLN, el movimiento campesino de Atenco y la comunidad indígena de Cherán en Michoacán. Por ejemplo, en el documento 2 denominado Posicionamiento Político previo a las elecciones presidenciales, el movimiento vuelve a definir sus objetivos de petición de imparcialidad en el proceso electoral del 2012 y también las relativos a la democratización de los medios de comunicación (Muñoz, 2011, p. 316). Las únicas menciones a temas relativos a políticas de identidad se refieren a la solidaridad con los esfuerzos de creación de comunidades autónomas en Cherán y Ostula y al declararse anti-neoliberal, el movimiento denuncia el despojo de las tierras y recursos de las comunidades originarias. ${ }^{2}$ Posteriormente en un Documento 3 denominado Plan de Lucha, el movimiento trata el tema de un cambio en el modelo educativo, científico y tecnológico se hace una apelación a una educación que entre otros adjetivos incluye paradójicamente tanto la pluriculturalidad como el humanismo (Muñoz, 2011, p. 319). La inclusión de ambos conceptos no solo resulta contradictoria, sino que si por educación humanista nos sujetamos al modelo occidentalizante/patriarcal, difícilmente puede ser compatible con la pluriculturalidad. En conclusión, el debate sobre inclusión étnico-racial se encontraba en un principio, fuera de la primera agenda del movimiento, que más bien se avocó a los temas de democratización y participación equitativa en medios.

1. Es el Centro Nacional de Evaluación para la Educación Superior, una asociación civil de carácter privado que tiene como objeto diseñar y aplicar pruebas de entrada y salida de la universidad y otros instrumentos de medición de desempeño en el ámbito universitario, su pagina web es:http://www.ceneval.edu.mx/

2. Documento 2. Posicionamiento previo a las elecciones presidenciales dado a conocer en la Ciudad de México el 28 de junio de 2012 del Movimiento Yo Soy 132 y Más de 133 en México. 
No obstante, de manera más reciente pero sin tanta visibilidad, el movimiento estudiantil Más de 131 incluyó acciones de solidaridad en asuntos puntuales relativos a movilizaciones de los pueblos indígenas. Estas tres acciones se pueden concretar en acciones de comunicación y acompañamiento en tres casos en particular: el décimo aniversario del experimento de organización autónoma de los cinco caracoles zapatistas en Chiapas, la presión para la liberación del Profesor Alberto Patishtán y la denuncia de la construcción de una carretera en el Estado de México dentro de un área natural protegida en territorio otomí.

La vinculación del movimiento estudiantil consu cobertura del evento de celebración del establecimiento de las formas de organización autónoma zapatista, muestra la gran simbiosis entre diferentes luchas sociales y el zapatismo. Una muestra es la solidaridad mostrada por el Ejercito Zapatista de Liberación $\mathrm{Na}$ cional y el Movimiento por la Paz con Justicia y Dignidad, que es la agrupación nacional más representativa de las victimas de la violencia reciente en México. Este último movimiento dictó un pronunciamiento a favor de la firma de los Acuerdos de San Andrés entre el Ejercito Zapatista de Liberación Nacional y el gobierno mexicano. Estos acuerdos cambiarían considerablemente la relación entre pueblos indígenas y el Estado mexicano. Por tanto, la solidaridad ejercida por el movimiento Más de 131, surgido a partir de la protesta contra la visita del ahora Presidente Enrique Peña Nieto a la Universidad Iberoamericana durante la campaña del 2012 y el Movimiento por la Paz con Justicia y Dignidad, muestran una clara tendencia de solidaridad hacia el modelo de autonomía indígena propuesto por el EZLN. Sin embargo, ninguna de las formas en las que el movimiento estudiantil Más de 131 se ha acercado a los movimientos indígenas muestra una exigencia por cambios en la configuración étnica, de género o de clase en las universidades públicas y privadas mexicanas.

A diferencia de los movimientos estudiantiles de la historia reciente mexicana, el movimiento estudiantil chileno incluyó dentro de su propuesta de cambio interno/externo una reivindicación de educación intercultural. Por ejemplo, la Confederación de Estudiantes de Chile (Confech), solicitó en una carta al Presiente Sebastián Piñera en 2011, una serie de 12 puntos referentes a la educación. Entre ellos se encuentra una amplía relación entre las exigencias de cambios en el sistema universitario y en el acceso a la educación en Chile, remarcando el doble carácter del movimiento de estudiantes chilenos, que incluye el cambio de la educación universitaria y una modificación estructural entre la sociedad, estado y educación. Dentro de las propuestas conocidas como los 12 puntos, la Confech incluía el reconocimiento de la educación como derecho social, la eliminación de la financiación bancaria de la misma así como el fin del lucro con la educación y término del sistema de financiación compartida. Se pueden englobar estas primeras demandas en una consigna de una reducción importante en la estratificación de clase de la educación, aunada a una petición más concreta que es la de generar nuevas formas de acceso a la Educación Superior. Un punto por demás interesante es que sumadas a estas exigencias de clase, la Confech incluyó dentro de su último punto una reivindicación educativa intercultural. Dicha punto se refería a una situación especialmente delicada, dada la conflictiva historia entre el Estado Chileno y el Pueblo Mapuche. Así los y las estudiantes solicitaban garantizar los derechos educativos y lingüísticos de los pueblos originarios bajo el Convenio 169 de la Organización Internacional del Trabajo y en la Ley indígena chilena 19.253. Es este el punto que muestra una solidaridad del movimiento estudiantil con las reivindicaciones de los pueblos indígenas, si bien limitándose a un área de su especial incidencia que es el educativo. Dicha relación no es solamente una basada en la solidaridad entre el movimiento estudiantil y los movimientos indígenas, sino que hace eco del ingreso dentro de la Confech, de la Federación Mapuche de Estudiantes y de su petitorio. Es en ese petitorio, que se hace referencia a la aplicación del Convenio 169 de la OIT y de la Ley 19.253, además de una propuesta genera por la interculturalidad. Quizá el punto que más llama la atención es la autodefinición de la federación de estudiantes no sólo como movimiento universitario, sino como un movimiento que: "lucha por la autodeterminación que escapa a los muros de la máquina de saber burgués y occidental que represente esta". En este sentido la petición de becas indígenas y modificación de las formas de entrada a la universidad, así como la creación de nuevos centros universitarios interculturales. La importancia del movimiento estudiantil chileno, se centra en que sus planteamientos tocan uno de los aspectos más consolidados del sistema político chileno, la privatización de la educación ideada por la dictadura y continuada durante la Concertación. Así la destrucción del carácter gratuito de la educación, que prevalecía hasta el golpe de Estado y el ataque constante al espíritu crítico en las ciencias sociales y humanidades, siguió durante el retorno de la democracia (Donoso Romo, 2005, p. 167). Por tanto el movimiento estudiantil chileno se muestra como una promesa en cuanto a la posibilidad de exigencia de una nueva relación entre la sociedad y el Estado chileno, que la vía democrática-electoral con los partidos tradicionales no pudo garantizar en por lo menos dos temas: educación y la relación con los pueblos indígenas.

Finalmente, por lo que respecta a las protestas estudiantiles puertorriqueñas, estas tienen un carácter 
de reivindicación étnica derivado de la situación colonial de Puerto Rico. En este sentido las diferentes formas de protesta de los y las estudiantes de la Universidad de Puerto Ricode 2010 a 2011 se dirigen hacia el intento de desmantelamiento neo-liberal de dicha universidad a través del establecimiento de medidas radicales de recortes y el establecimiento de una cuota a los alumnos. Como muestra de las paradojas de la universidad como sistema de perpetuación y también foco de disidencia, este movimiento se centró en la permanencia de una institución que le era incomoda políticamente al sistema político colonial en Puerto Rico. La forma de organización de los y las estudiantes puertorriqueñas mostraba la aplicación de una democracia radical mediante la formación de comités que no solo representaban la división tradicional entre facultades, sino que también se formaban comités en función de diversidad de género: de mujeres y de la comunidad gay, lesbiana, transgénero y bisexual (Atiles-Osoria, 2013, p. 110). Tanto las experiencias de las huelgas y movilizaciones en Chile, Puerto Rico y México, muestran como la universidad no solo se reduce a un legitimador de las élites, sino que también es un lugar de encuentro y politización. Sin embargo, el embate a la Universidad como lugar de discusión de cambios políticos se muestra más claro que nunca. Las limitaciones en el acceso y la sobre burocratización, son partes importantes de estas estrategias. Solo cabe proponer algunas formas en que la Universidad como lugar de encuentro político puede sobrevivir dicho embate.

\section{Dos opciones radicales: interculturalizar y desescolarizar la Universidad}

Quizá la Universidad Latinoamericana como institución apropiada por el neoliberalismo se ha convertido como señala Iván Illich como: "el recinto encargado de la custodia de quienes sobran en la calle, el hogar o el mercado laboral" (Illich, 2006, p. 107). De hecho la creciente criminalización de la juventud en los centros urbanos en América Latina, parece dejar la administración de los cuerpos de los y las jóvenes a sólo dos instituciones del biopoder: la penitenciaria o la escolar. La diferencia radica en que una se percibe como la última instancia de control social y como una caja negra en laque depositar los elementos que la sociedad no quiere tener en su rango de mirada, mientras que la otra institución se vende como el vehículo o llave a la promoción directa de movilidad de clase socio-económica. El establecer a la universidad como instrumento representativo de la meritocracía, sería una forma más de mantener el esquema de dominación de una élite egresada de dichos centros, que incluye, en palabras otra vez de Ivan Illich: "los líderes políticos y los técnicos de planificación, independientemente de que sean conservadores, marxistas o liberales" (Illich, 2006, p. 106). El carácter emancipatorio de la educación en general y de la universidad en particular queda bastante en entredicho, sobre todo debido a que en muchos casos las protestas tendientes a reafirmar el derecho a la educación provienen de quienes fueron excluidos o cuyo derecho fue firmemente limitado a acceder a la educación superior. Pensemos en los estudiantes/ deudores de excesivos créditos educativos en Chile o Colombia o las protestas de los "no-admitidos" a los sistemas de educación pública superior en México. Aquí el sistema de exclusión se divide entre los educados y los no educados y por ende la entrada a la universidad se convierte en un claro barómetro social de accesibilidad al ascenso social o de perpetuación del privilegio social. En este sentido Esteva, Prakash y Stuchul, realizan una fuerte crítica a Paulo Freire, según la cual la división entre educados/no educados instaura un sistema de exclusión bajo la cual las minorías educadas obtienen grandes privilegios de dicha condición (Esteva, Prakash y Stuchul, 2004, p. 13). Volviendo a Iván Illich: "La escuela, que ayudó en el siglo pasado a superar el feudalismo, se está convirtiendo en ídolo opresor que sólo protege a los escolarizados" (Illich, 2006, p. 110). Continúa diciendo: "La aguda pirámide educacional asigna a cada individuo su nivel de poder, prestigio y recursos, según considera apropiado para él"(Illich, 2006, p. 111).

Quizá la propuesta de desescolarización de Illich no es tan radical como parece, mudando el centro del esfuerzo material de las universidades a otras formas de educación superior. De hecho, en el ámbito indígena hubo un esfuerzo de desescolarización, debido a que el sistema educativo creaba amplías fracturas entre los alumnos y alumnas y sus comunidades, ya sea a través de la pérdida de generaciones debido a su permanencia en las ciudades, o la desindianización, o mestizaje criollizante operado desde la universidad. Las "oportunidades" de ascenso social que les permite la incorporación universitaria es el acceso a empleos no profesionales en la ciudad y una formación que ni logra vencer el racismo estructural del mercado laboral urbano, ni el desarrollo de capacidades aplicadas en su comunidad (Esteva, 2007).

Quizá aquí la opción es reinterpretar el paradigma de Freire (2008) de la pedagogía del oprimido, los oprimidos en este caso las comunidades indígenas ayudarían no sólo a su liberación sino a la liberación de los opresores, en este caso a los ladinos o mestizos respecto de la educación eurocéntrica y patriarcal. La opción, que en cierta medida haría eco de las deman- 
das estudiantiles sería la transformación de la universidad en sí misma. Quizá inclusive con fórmulas que no solo se refieran a la creación de universidades inter/ multiculturales que se insertan más en el modelo de neoliberalismo multicultural, sino interculturalizar a las universidades existentes. Así como las exigencias estudiantiles en San Francisco llevaron a un replanteamiento en la configuración racial de las universidades norteamericanas, así los puntos petitorios de los estudiantes chilenos en materia intercultural y la organización de Comités en función de diversidad de género en las protestas de la Universidad de Puerto Rico, pueden prometer formas de modificación del sistema educativo. Sin embargo, la opción debe ser por desestabilizar la división entre escolarizados y no escolarizados, como la noción de ciencia u poder/saber por el de saberes. Las experiencias desde los modelos de universidades obreras, campesinas e indígenas, así como los departamentos de extensión universitaria deben ser vistas en todos sus triunfos y derrotas. Se debe evitar crear lugares aislados dentro de las universidades que no sólo se encuentren separadas del resto de la estructura, sino que sean blancos fáciles de los recortes y modificaciones neoliberales. También los gruesos muros de accesibilidad y de separación social, así como las formas autoritarias y autocomplacientes de la academia, deben de dar paso a aprender de personas que no tienen un estatuto de educadores o educadores oficiales y acercarse a personas que no tienen una categoría de estudiantes reconocidos.

\section{Referencias}

ALMADA, M. Paraguay: educación y dependencia. Asunción: Marben, 2013.

ANDERSON, R.; GONZALEZ M. T.; LEE, S. Toward a new student insurgency: a critical epistolary. California Law Review, v. 94, n. 6, p. 1879-1946, Dec. 2006.

ATILES-OSORIA, J. M. Neoliberalism, law and strikes: law as an instrument of repression at the University of Puerto Rico, 2010-2011. Latin American Perspectives, v. 40, n. 5, p. 105-117, Sep. 2013.

BARRIOS, J. L. Del nombre y el síntoma: Yo soy \#131/ Yo soy \#132. Campus Expandido, Museo Universitario de Arte Contemporáneo de la UNAM, Junio de 2012, disponible en: <http://www.muac.unam.mx/ proyectos/campusexpandido/paralelas/18.html>.

BERGER, D. Outlaws of America: the weather underground and the politics of solidarity. Oakland: AK Press, 2005.

DONOSO ROMO, A. Exclusiones en la educación superior chilena: preguntas abiertas para el siglo XXI. Revista de Sociología, n. 19, p. 159-170, 2005.

ECHEVERÍA, B. Modernidad y blanquitud. México: Era, 2010

ESTEVA, G. Reclaiming our Freedom to Learn. YES! Magazine, noviembre v. 7, 2007. Disponible en: <http://www.yesmagazine.org/issues/liberate-yourspace/reclaiming-our-freedom-to-learn $>$.

ESTEVA, G.; PRAKASH, M. S.; STUCHUL, D. L. From a pedagogy for liberation to liberation form pedagogy. In: BROWERS, C. A.; APFFELMARGLIN, F. Rethinking Freire: globalization and the environmental crisis. Mahwa: Lawrence Erlbuam, Associates, 2005.

FREIRE, P. Pedagogía del oprimido. 58. ed. México: Siglo XXI, 2008.

HALE, C. R. Neoliberal multiculturalism. PoLAR: Political and Legal Antropology Review, v. 28, n. 1, p. 10-19, may 2005.

ILLICH, I. Obras reunidas, v. I. México: FCE, 2006. JUDT, T. The memory Chalet. Londres: Penguin, 2010. MUÑOZ RAMÍREZ, G. (Coord.). \#Yo Soy132: voces del movimiento. México: Bola de Cristal, 2011. ROTHBART, G. S. The legitimation of inequality: objective scholarship vs black militance. Sociology of Education, v. 43, n. 2, p. 159-171, Spring 1970.

VIEYRA, J. Tres perspectivas sobre el pluralismo cultural en México (Vasconcelos, León Portilla, Bonfil Batalla). In: KLEISING-REMPEL, U. (Comp.). Lo propio y lo ajeno: interculturalidad y sociedad multicultural. México: Plaza y Valdés, 1996. p. 51-72. 


\title{
Interculturalidade, universidade e movimentos sociais latino-americanos:
}

\section{ideias desde a fronteira do norte}

\section{Resumo}

A ascensão social, por meio da educação superior parece uma promessa difícil de cumprir na paisagem atual da América Latina. A educação bancária, mencionada nas críticas de Freire e Illich, está aqui para ficar, deixando um paradoxo aberto entre a Universidade-Usina e a Universidade como um lugar de politização. O que fica da luta pela inclusão de sujeitos subalternos às universidades? Para que integrar? A proposta subjacente, para resolver este paradoxo, pressupõe não só incluir, mas interculturalizar e descolonizar a universidade.

Palavras-chave: educação superior, movimentos estudantis, educação intercultural, educação na América Latina.

\section{Interculturality, universities and Latin American social movements: ideas}

\section{from the border to the north}

\begin{abstract}
The promise of social ascent trough higher education seems almost impossible to fulfill in today's Latin America. It seems like the Freire's and Illich's concept of banking education has come to stay. This in turn creates an open paradox between the university as a factory and the university as a place for politics. What is left of the struggle for inclusion of the subaltern into the university? What is the final purpose of integration? The answer lies within the confrontation of this paradox, not only must the university be inclusive, but it must become intercultural and decolonized.
\end{abstract}

Key words: higher education, student movements, intercultural education, education in Latin America.

Data de recebimento do artigo: 13/5/2013

Data de aprovação do artigo: 20/12/2013 